\title{
Análisis de resultados de ensayos de resistencia de probetas de tierra
}

\section{Analysis of results of tests carried out on earth test pieces}

\author{
JULIAN SALAS, Dr. Ing. Industrial (IETcC-CSIC) \\ MARIA JESUS GUINEA, Arquitecta (IETCC-CSIC) \\ Serrano Galvache, s.n. 28033 Madrid/España \\ Fecha de recepción: 15-IV-88 \\ JUAN DIEGO BERNAL, Arquitecto (MEDELLIN-Colombia) (*)
}

\section{RESUMEN}

Partiendo de un suelo concreto, tierra roja de Soria, de la que se aportan datos para su caracterización físico-química, se han realizado series de probetas de $4 \times 4 \times 16 \mathrm{~cm}$, sobre las que se ha estudiado la influencia: del aumento de cemento sobre distintas proporciones tierra/arena; del tipo de cemento; de la variación de la proporción de cemento sobre relaciones concretas tierra/arena; ...presentando en todos los casos los valores a compresión, a flexotracción, asi como la interrelación entre ellos.

\section{SUMMARY}

A series of test pieces of $4 \times 4 \times 16$ were prepared from Red Earth from Soria, the physical-chemical characteristics of which are given. The effect of each of the following upon the test pieces was studied: increasing the amount of cement on different earth/sand proportions; the type of cement; the variation of the cement proportion maintaining constant earth/sand proportions. In each case the bending-tensile and compressive strengths are given, and well as interrelationships between them.

\section{PALABRAS CLAVE:}

Tierra; Ensayos; Consolidación; Resistencias mecánicas.

\section{CARACTERISTICAS DE LOS MATERIALES EMPLEADOS}

\subsection{Suelo rojo de Soria}

El suelo empleado queda definido, a efectos de este trabajo, por la aportación de resultados de las siguientes pruebas: Análisis mineralógico por difracción de Rayos $X$ (Fig. 1); Composición química (Tabla 1); Estudio granulométrico (Figs. $2 a$ y $2 b$ ) y Características geotécnicas y mecánicas (Tabla 2)

(*) Este trabajo, realizado en el marco del Equipo de investigación "Viviendas de muy bajo coste", es resultado de un dilatado proceso de ensayos, en parte de los cuales se contó con la colaboración de Edgard Rohmer y Carmen Luengo. Igualmente han aportado su inestimable colaboración las Dras. Luxán y Sanchez-Rojas así como el Dr. Sagrera y la Lda. Gómez. A todos ellos nuestro agradecimiento.
KEY WORDS:

Earth; Tests; Consolidation; Mechanical Strengths.

\section{CHARACTERISTICS OF THE MATERIALS EMPLOYED}

\subsection{Red Earth from Soria}

The earth used is defined for the purposes of this study by the results of the following tests: mineralogical analysis by $X$-ray diffraction (Fig. 1); chemical composition (Table 1); particle size analysis (Figs. $2 a$ and $2 b$ ) and geo-technical and mechanical characteristics (Table 2).

(*) This study, carried out within the framework of the Research Team "Very Low Cost Housing", is the result of a long series of tests, some of which saw the collaboration of Edgard Rohmer and Carmen Luengo. Likewise, we are indebted to Drs. Luxán and Sanchez-Rojas, as well as Dr. Sagrera and Ms. Gómez for their invaluable collaboration. 
$\times 10^{4}$

1.90

5.81

0.64

5.49

0.36

Q.25 -

0.16

0.09

0.04

0.91

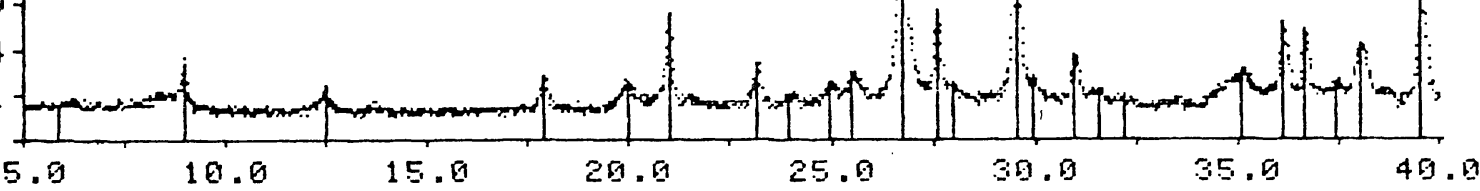

0.31

0.64

9.49

0.36

0.25

0.16

0.09

0.94

0.01

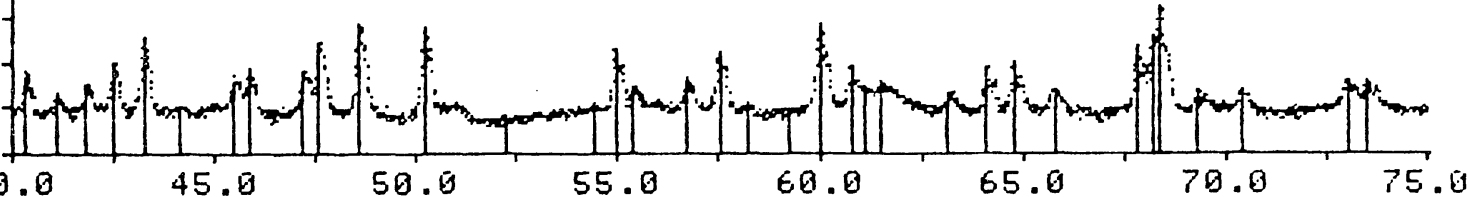

Fig. 1.-Difracción de Rayos X.

Fig. 1.-X-ray diffraction.

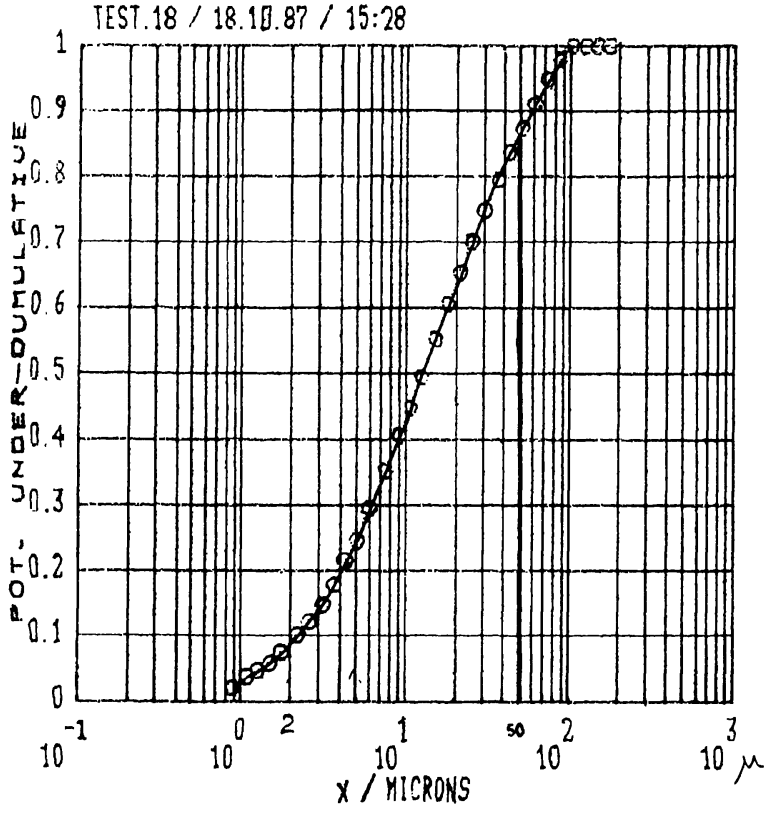

Fig. 2a.-Curva granulométrica.

Fig. 2a.-Granulometric graphic

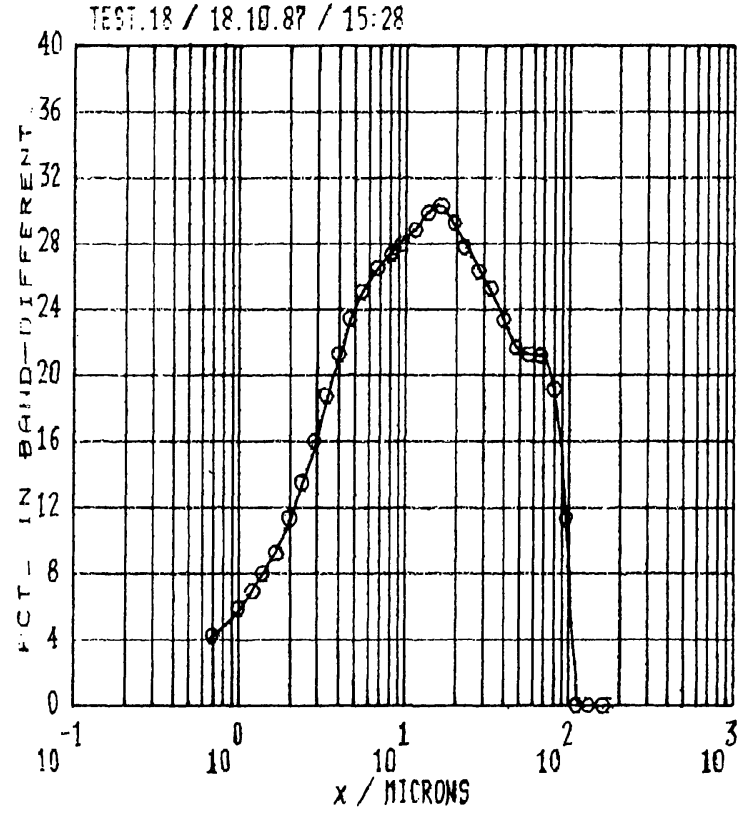

Fig. 2b.-Densidades de concentración.

Fig. 2b.-Densities of concentration. 
TABLA 1 (TABLE 1)

Composición química del Suelo Rojo de Soria (Chemical composition of Red Earth from Soria)

\begin{tabular}{|l|r|}
\hline \multicolumn{1}{|c|}{ ANALISIS QUIMICO (CHEMICAL ANALYSIS) } & $\%$ \\
\hline Pérdida por calcinación a $1.100^{\circ} \mathrm{C}$ (Loss through burning at $1.100^{\circ} \mathrm{C}$ ) & 18,6 \\
Sílice (Silica), $\mathrm{SiO}_{2}$ & 47,9 \\
Oxido de Hierro (Ferric Oxide), $\mathrm{Fe}_{2} \mathrm{O}_{3}$ & 2,1 \\
Oxido de Titanio (Titanium Oxide), $\mathrm{TiO}_{2}$ & 0,3 \\
Oxido de Aluminio (Aluminum Oxide), $\mathrm{Al}_{2} \mathrm{O}_{3}$ & 8,5 \\
Oxido de Magnesio (Magnesium Oxide), $\mathrm{MgO}$ & 1,3 \\
Oxido de Calcio (Calcium Oxide), $\mathrm{CaO}$ & 19,2 \\
Oxido de Sodio (Sodium Oxide), $\mathrm{Na}{ }_{2} \mathrm{O}$ & 0,1 \\
Oxido de Potasio (Potassium Oxide), $\mathrm{K}_{2} \mathrm{O}$ & 1,6 \\
\hline
\end{tabular}

TABLA 2 (TABLE 2)

Caraterísticas geotécnicas y mecánicas del Suelo Rojo de Soria

(Geotechnical and Mechanical Characteristics of Red Earth from Soria)

\begin{tabular}{|c|c|}
\hline VALORES CUANTITATIVOS (QUANTITATIVE VALUES) & Suelo Rojo \\
\hline Determinación de finos (Determination of fine particles) $(<80$ UNE-7135) & 63,10 \\
\hline Contenido en arcilla (Clay content) $(<2 \%)$ & 26,60 \\
\hline Humedad natural (en laboratorio) [Natural humidity (in laboratory)] (\%) & 2,41 \\
\hline Indice de huecos (Cavity or hollow index) $(e=2,65$ w) & 0,06 \\
\hline Porosidad (Porosity) $(n=e / 1-e)$ & 0,60 \\
\hline Densidad seca (Dry density) $\gamma_{d}=\gamma_{n}(1+w)$ & 0,37 \\
\hline Límite plástico (Plasticity Limit) (\%) LP $(<0,40 \mathrm{~mm})$ & 24,10 \\
\hline Límite líquido (Liquid Limit) (\%) LL $(<0,40 \mathrm{~mm})$ & 38,50 \\
\hline Indice plástico (Plasticity Index) IP & 14,40 \\
\hline Actividad (Activity) (IP/arcilla) & 0,54 \\
\hline Retracción (Retraction) (\%) & 5,20 \\
\hline Resistencia al agua (Water resistance) (en 1 hora) & válido \\
\hline Resistencia a tracción (prueba de los ochos) [Tensile strength (the 8's test)] (kp/ $\left.\mathrm{cm}^{2}\right)$ & 2,75 \\
\hline Resistencia a compresión (Compressive strength) $\left(\mathrm{kp} / \mathrm{cm}^{2}\right) 4 \times 4 \times 16 \mathrm{~cm}$ & 67,50 \\
\hline Resistencia a flexión (Bending Strength) $\left(\mathrm{kp} / \mathrm{cm}^{2}\right) 4 \times 4 \times 16 \mathrm{~cm}$ & 23,50 \\
\hline
\end{tabular}

En la figura 3 se reproduce el ábaco de clasificación de suelo por su textura. La zona rayada representa los suelos aptos para la construcción con tierra. El suelo empleado en este trabajo presenta las siguientes proporciones:

Arena: 36,9\%; Limo: 36,5\%; Arcilla: 26,6\%

A la vista del ábaco, se deduce que el suelo es apto para la estabilización y la construcción. Podría objetarse la presencia de un exceso $(63,1 \%)$ de finos (limo+arcilla), por lo tanto ha de corregirse la granulometría, añadiendo una pequeña proporción de arena, consiguiendo así una granulometría no uniforme $y$, por
Figure 3 shows the abacus of the classification of the earth by texture. The striped zone represents the soils apt for earthen construction. The earth used in this study yields the following proportions:

Sand: $36.9 \%$; $\quad$ Mud: $36.5 \% ; \quad$ Clay: $26.6 \%$

It can be deduced from this abacus that the earth is apt for stabilizing and construction. The presence of an excess of fine particles (mud + clay $=63.1 \%$ ) can be objected to; thus the graduation must be corrected by adding a small proportion of sand. In this way a non-uniform graduation is 
consiguiente, una compactación más eficaz (1) y (2).

Según L. Holmgren (3), el coeficiente de uniformidad modificado (c.u.m.) debe estar comprendido entre 8 y 70 , siendo dicho coeficiente el cociente entre el diámetro por el que pasan un $80 \%$ de las partículas y el diámetro por el que pasan el $60 \%$. El suelo empleado presenta un c.u.m. de valor 8,0 y los diámetros respectivos son $0,4 \mathrm{~mm}$ y $0,5 \mathrm{~mm}$.

Respecto a la retracción de la tierra empleada y adoptando la relación entre el IP y la retración lineal según el diagrama del State Highway Laboratory (USA) (2):

Retracción lineal $=0,654 \mathrm{IP}-0,00333 \mathrm{IP}^{2}$.

Para el suelo en estudio: IP $=14,4$;

por lo tanto la R.L. $=8,73 \%$.

La retracción no debe ser superior al $3 \%$ ni inferior al 0,3\%, por lo que resulta necesario añadir algo de arena para rebajar la alta retracción lineal.

Según el cuadro de zonas preferenciales de estabilización, en función de la plasticidad (Fig. 4), el suelo estudiado se sitúa en la zona 6 , por lo que resulta aconsejable estabilizar con cemento.

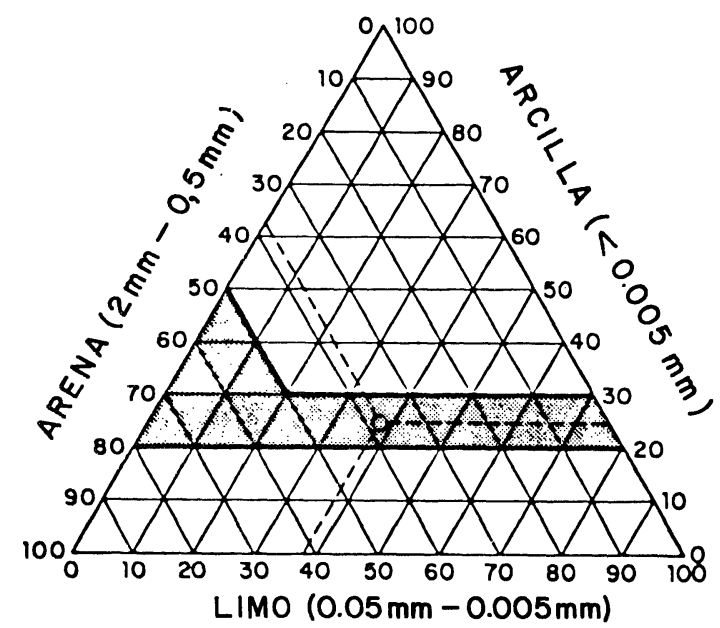

Fig. 3.-Abaco de clasificación de los suelos por su textura.

Fig. 3.-Soils clasification abacus by textures. achieved and, therefore, more effective compacting (1) and (2).

According to L. Holmgren (3), the Modified Uniformity Coefficient (CUM) should range between 8 and 70. Said coefficient is the quotient between the diameter through which pass $80 \%$ of the particles and the diameter through which $60 \%$ pass. The earth employed has a CUM of 8.0, and the respective diameters are $0.4 \mathrm{~mm}$ and $0.5 \mathrm{~mm}$.

With respect to the retraction (shrinkage) of the earth used, and adopting the relation between the Plasticity Index (PI) and the linear retraction (L.R.), according to the diagram of the State Highway Dept., U.S.A. (2):

Linear Retraction $=0.654 \mathrm{PI}-0.00333(P I)^{2}$

For the earth under study: $P I=14.4$; therefore, the L.R. $=8.73 \%$.

The retraction should not be superior to $3 \%$, nor inferior to $0.3 \%$; therefore it is necessary to add some sand in order to reduce the high linear retraction.

According to the chart of preferential stabilization zones in terms of plasticity (fig. 4), the aerth under study belongs in zone 6 , which makes it advisable to stabilize with cement.

ZONAS PREFERENCIALES DE ESTABILIZACION EN FUNCION DE LA PLASTICIDAD

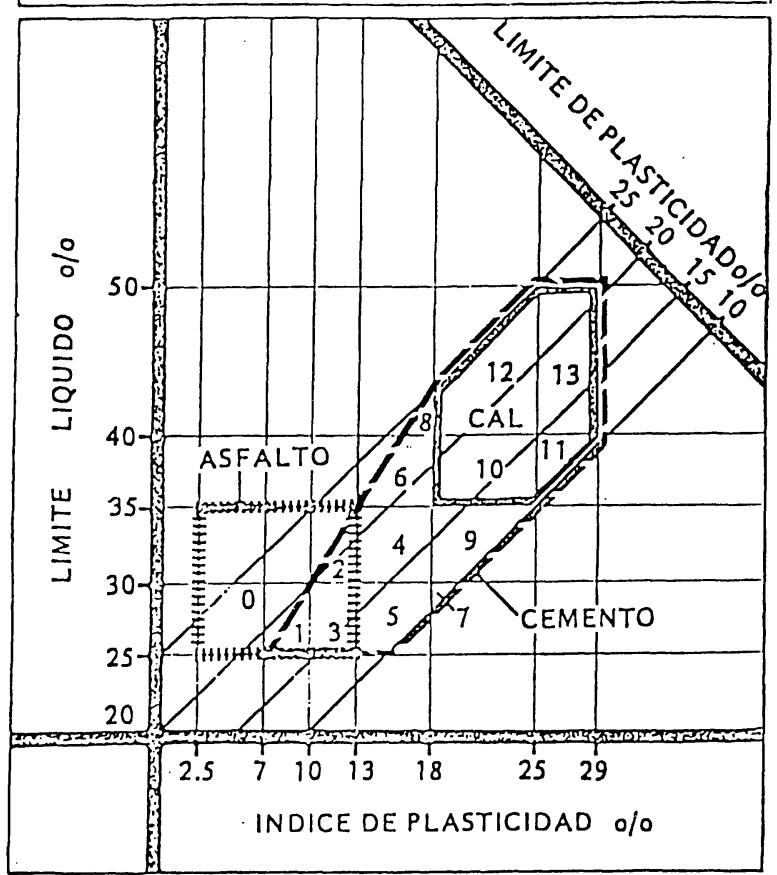

Fig. 4.-Cuadro de zonas preferenciales de estabilización en función de la plasticidad.

Fig. 4.-Preferential zones of stabilization by plasticity.

MATERIALES DE CONSTRUCCION, Vol. 38, n.०210, abril/mayo/junio 1988 
El suelo empleado tiene una composición mineralógica que contiene: cuarzo, calcita, ortosa, minerales micáceos, minerales kandíticos (caolinita) y clorita o vermiculita.

\subsection{Arena normalizada}

En la Tabla 3 se recogen las características granulométricas de la arena empleada.
The mineralogical composition of the earth studied is the following: quartz, calcite, orthoclose, micaceous minerals, kanditic minerals (calonite), and chlorite or verniculite.

\subsection{Standardized Sand}

The graduation of the sand used are shown in Table 3.

TABLA 3 (TABLE 3)

Granulometría de la arena empleada (Graduation of the sand used)

\begin{tabular}{|c|c|c|c|}
\hline $\begin{array}{c}\text { TAMIZ } \\
\text { SIEVE }\end{array}$ & $\begin{array}{c}\text { MALLA } \\
\text { MESH }\end{array}$ & $\begin{array}{c}\text { LIMITES G } \\
\text { G LIMITS }\end{array}$ \\
\hline 1 & 2,000 & 0 & $6-16$ \\
2 & 1,500 & $90-240$ & $18-28$ \\
3 & 1,200 & $270-420$ & $42-52$ \\
4 & 0,750 & $630-780$ & $60-72$ \\
6 & 0,500 & $900-1.080$ & $74-84$ \\
7 & 0,250 & $1.110-1.260$ & $83-93$ \\
9 & 0,150 & $1.245-1.395$ & $93-98$ \\
\hline
\end{tabular}

TABLA 4 (TABLE 4)

\begin{tabular}{|l|r|}
\hline \multicolumn{1}{|c|}{ ANALISIS QUIMICO PA-350 (CHEMICAL ANALYSIS PA-350) } & $\%$ \\
\hline Pérdida por calcinación (Loss by Burning) (P.F.) & 2,91 \\
Residuo insoluble (Insoluble Residue) (R.I.) & 2,73 \\
Sílice (Silica), $\mathrm{SiO}_{2}$ & 22,87 \\
Oxido de Aluminio, (Aluminum Oxide), $\mathrm{Al}_{2} \mathrm{O}_{3}$ & 6,00 \\
Oxido de Hierro (Ferric Oxide), $\mathrm{Fe}_{2} \mathrm{O}_{3}$ & 4,00 \\
Oxido de Calcio (Calcium Oxide), $\mathrm{CaO}$ & 53,84 \\
Oxido de Magnesio (Magnesium Oxide), $\mathrm{MgO}^{-3,60}$ \\
Anhidrido Sulfúrico (Sulfuric Anhydride), $\mathrm{SO}_{3}$ & 2,90 \\
\hline
\end{tabular}

TABLA 5 (TABLE 5)

\begin{tabular}{|l|r|}
\hline \multicolumn{1}{|c|}{ ANALISIS QUIMICO ARI-350 (CHEMICAL ANALYSIS ARI-350) } & $\%$ \\
\hline Pérdida por calcinación (Loss by Burning) (P.F.) & 2,45 \\
Residuo insoluble (Insoluble Residue) (R.I.) & 1,84 \\
Sílice (Silica), $\mathrm{SiO}_{2}$ & 19,85 \\
Oxido de Aluminio, (Aluminum Oxide), $\mathrm{Al}_{2} \mathrm{O}_{3}$ & 5,88 \\
Oxido de Hierro (Ferric Oxide), $\mathrm{Fe}_{2} \mathrm{O}_{3}$ & 3,17 \\
Oxido de Calcio (Calcium Oxide), $\mathrm{CaO}$ & 61,32 \\
Oxido de Magnesio (Magnesium Oxide), $\mathrm{MgO}$ & 1,51 \\
Anhídrido Sulfúrico (Sulfuric Anhydride), $\mathrm{SO}_{3}$ & 3,00 \\
\hline
\end{tabular}




\subsection{Cemento PA-350}

En la Tabla 4 se recoge la composición química del cemento empleado; se trata de un cemento portland con hasta un $19 \%$ de adiciones.

\subsection{Cemento ARI-450}

En la Tabla 5 se recoge la composición química del cemento ARI-450.

\section{PREPARACION DE LAS PROBETAS}

\subsection{Mezcla de componentes}

El suelo se preparó antes de la mezcla en la forma siguiente:

a) se secó en estufa a $60^{\circ} \mathrm{C}$ y $0 \%$ H.R. Se realizó esta operación para poder calcular con fiabilidad el agua de amasado, ya que ésta depende considerablemente de la humedad natural que retiene el suelo, debido a la arcilla;

b) se machacó con martillo de goma, para desmenuzar los terrones;

c) se pasó por el tamiz de $2.000 \mu \mathrm{m}$; n. ${ }^{\circ} 10$, UNE-7050.

La mezcla se mantuvo durante 10 minutos en una túrbula Tipo T-2C, Nr. 810820, para conseguir mayor homogeneidad, se agregó a continuación el agua y se amasó manualmente durante 5 minutos como mínimo, para conseguir un reparto lo más homogéneo posible de la misma. Las probetas se realizaron en moldes de tres, de dimensiones $4 \times 4 \times 16$ centímetros.

La masa se colocó en tres tongadas, golpeando a mano con una maza de madera para conseguir una cierta compacidad, comprobándose que, aun siendo manual, es éste un factor claramente influyente sobre la porosidad y la resistencia mecánica de las probetas.

\subsection{Aclaración sobre valores porcentuales}

Por razones de claridad metodológica, en todos los casos se ha optado por mantener constantes las proporciones porcentuales de los materiales componentes de las probetas ensayadas, aún conscientes de que el planteamiento matemático es erróneo. En la Tabla 6 se recogen las equivalencias porcentuales reales. Por ejemplo, cuando nos referimos a probetas con "un $80 \%$ de tierra, $20 \%$ de arena normalizada y $12 \%$ de

\subsection{PA-350 Cement}

The chemical analysis of the cement used is shown in Table 4. It is Portland cement with up to $19 \%$ additions.

\subsection{ARI-450 Cement}

The chemical analysis of the ARI-450 cement used is shown in Table 5.

\section{PREPARATION OF TEST PIECES}

\subsection{Component Mix}

Before mixing, the earth was prepared in the following manner:

a) stove-dried at $60^{\circ} \mathrm{C}$ and $0 \%$ R.H. This was done in order to accurantely determine the mixing water, since this depended considerably on the natural humidity retained by the earth's clay content;

b) crushed using a rubber hammer in order to break up clods;

c) passed through a sieve of $2.000 \mu \mathrm{m}$; no. 10 , UNE-7050.

The mix was kept for 10 minutes in a type T-2C, Nr. 810820 mixer in order to achieve greater homogeneity; later, water was added and the mix was manually mixed for at least 5 minutes in order to achieve the greatest homogeneity possible. The test pieces were executed in molds of three, with dimensions of $4 \times 4 \times 16 \mathrm{~cm}$

The mass was then set in three layers, manually struck with a wooden maul in order to compact it somewhat. This factor, even if acheved manually, clearly influences the porosity and the mechanical strength of the test pieces.

\subsection{Note on Percentage Values}

In each case, and in order to follow a clear-cut methodology, we have chosen to maintain constant the percentages of test pieces component materials, despite being aware that mathematically this is erroneous. Table 6 shows the real percentage equivalents. For example, when we refer to test pieces of " $80 \%$ earth, $20 \%$ standardized sand and $12 \%$ cement" one must understand the real percentage values to be, according to said 
TABLA 6 (TABLE 6)

Valores reales porcentuales, en función de los tantos por ciento teóricos (Real percentage values, in terms of theoretical percentage points)

\begin{tabular}{|c|c|c|c|c|c|c|c|c|c|c|}
\hline \multirow{2}{*}{$\begin{array}{l}\% \text { Tierra } \\
\text { (\% Earth) } \\
\% \text { Arena } \\
\text { (\% Sand) }\end{array}$} & \multicolumn{10}{|c|}{$\%$ CEMENTO (\% CEMENT) } \\
\hline & 2 & 4 & 6 & 8 & 10 & 12 & 14 & 16 & 18 & 20 \\
\hline $\begin{array}{r}100 \\
0\end{array}$ & $\begin{array}{l}98,03 \\
0 \\
1,97\end{array}$ & $\begin{array}{l}96,15 \\
0 \\
3,85\end{array}$ & $\begin{array}{l}94,33 \\
0 \\
5,67\end{array}$ & $\begin{array}{l}92,59 \\
0 \\
7,41\end{array}$ & $\begin{array}{l}90,90 \\
0 \\
9,1\end{array}$ & $\begin{array}{c}89,28 \\
0 \\
10,72\end{array}$ & $\begin{array}{c}87,21 \\
0 \\
12,29\end{array}$ & $\begin{array}{c}86,20 \\
0 \\
13,80\end{array}$ & $\begin{array}{c}84,74 \\
0 \\
15,26\end{array}$ & $\begin{array}{c}83,33 \\
0 \\
16,67\end{array}$ \\
\hline $\begin{array}{r}90 \\
0\end{array}$ & $\begin{array}{c}88,23 \\
9,8 \\
1,97\end{array}$ & $\begin{array}{r}86,53 \\
9,61 \\
3,86\end{array}$ & $\begin{array}{r}84,90 \\
9,2.5 \\
5,49\end{array}$ & $\begin{array}{r}83,33 \\
9,10 \\
7,42\end{array}$ & $\begin{array}{r}81,80 \\
9,10 \\
9,10\end{array}$ & $\begin{array}{r}80,35 \\
8,92 \\
10,73\end{array}$ & $\begin{array}{r}78,94 \\
8,77 \\
17,29\end{array}$ & $\begin{array}{r}77,58 \\
8,62 \\
13,80\end{array}$ & $\begin{array}{r}76,27 \\
8,47 \\
15,26\end{array}$ & $\begin{array}{r}75,00 \\
8,33 \\
16,67\end{array}$ \\
\hline $\begin{array}{l}80 \\
20\end{array}$ & $\begin{array}{r}78,43 \\
19,61 \\
1,96\end{array}$ & $\begin{array}{r}76,92 \\
19,23 \\
3,85\end{array}$ & $\begin{array}{r}75,47 \\
18,86 \\
5,67\end{array}$ & $\begin{array}{r}74,07 \\
18,52 \\
7,41\end{array}$ & $\begin{array}{r}72,72 \\
18,18 \\
9,10\end{array}$ & $\begin{array}{l}71,43 \\
17,86 \\
10,71\end{array}$ & $\begin{array}{l}70,17 \\
17,54 \\
12,28\end{array}$ & $\begin{array}{l}68,96 \\
17,24 \\
13,80\end{array}$ & $\begin{array}{l}67,79 \\
16,95 \\
15,26\end{array}$ & $\begin{array}{l}66,66 \\
16,67 \\
16,67\end{array}$ \\
\hline $\begin{array}{l}70 \\
30\end{array}$ & $\begin{array}{r}68,62 \\
29,41 \\
1,97\end{array}$ & $\begin{array}{r}67,30 \\
28,84 \\
3,86\end{array}$ & $\begin{array}{r}66,03 \\
28,30 \\
5,67\end{array}$ & $\begin{array}{r}64,81 \\
27,77 \\
7,42\end{array}$ & $\begin{array}{r}63,63 \\
27,27 \\
9,10\end{array}$ & $\begin{array}{l}62,50 \\
26,78 \\
10,72\end{array}$ & $\begin{array}{l}61,40 \\
26,31 \\
12,29\end{array}$ & $\begin{array}{l}60,34 \\
25,86 \\
13,80\end{array}$ & $\begin{array}{l}59,32 \\
25,42 \\
15,26\end{array}$ & $\begin{array}{l}58,33 \\
25,00 \\
16,67\end{array}$ \\
\hline $\begin{array}{l}60 \\
40\end{array}$ & $\begin{array}{r}58,72 \\
39,21 \\
1,97\end{array}$ & $\begin{array}{r}57,69 \\
38,46 \\
3,85\end{array}$ & $\begin{array}{r}56,60 \\
37,73 \\
5,67\end{array}$ & $\begin{array}{r}55,55 \\
37,03 \\
7,42\end{array}$ & $\begin{array}{r}54,54 \\
36,35 \\
9,10\end{array}$ & $\begin{array}{l}53,57 \\
35,71 \\
10,72\end{array}$ & $\begin{array}{l}52,63 \\
35,08 \\
12,29\end{array}$ & $\begin{array}{l}51,72 \\
34,48 \\
13,80\end{array}$ & $\begin{array}{l}50,84 \\
33,89 \\
15,27\end{array}$ & $\begin{array}{l}50,00 \\
33,33 \\
16,67\end{array}$ \\
\hline $\begin{array}{l}50 \\
50\end{array}$ & $\begin{array}{r}49,01 \\
49,01 \\
1,98\end{array}$ & $\begin{array}{r}48,07 \\
48,07 \\
3,86\end{array}$ & $\begin{array}{r}47,16 \\
47,16 \\
5,68\end{array}$ & $\begin{array}{r}46,29 \\
46,29 \\
7,42\end{array}$ & $\begin{array}{r}45,45 \\
45,45 \\
9,10\end{array}$ & $\begin{array}{l}44,64 \\
44,64 \\
10,72\end{array}$ & $\begin{array}{l}43,85 \\
43,855 \\
12,30\end{array}$ & $\begin{array}{l}43,10 \\
43,10 \\
13,80\end{array}$ & $\begin{array}{l}42,37 \\
43,27 \\
15,26\end{array}$ & $\begin{array}{l}41,66 \\
41,66 \\
17,68\end{array}$ \\
\hline $\begin{array}{l}40 \\
60\end{array}$ & $\begin{array}{r}39,21 \\
58,82 \\
1,97\end{array}$ & $\begin{array}{r}38,46 \\
57,69 \\
3,85\end{array}$ & $\begin{array}{r}37,73 \\
56,60 \\
5,67\end{array}$ & $\begin{array}{r}37,03 \\
55,55 \\
7,42\end{array}$ & $\begin{array}{r}36,36 \\
54,54 \\
9,10\end{array}$ & $\begin{array}{l}35,71 \\
53,57 \\
10,72\end{array}$ & $\begin{array}{l}35,08 \\
52,63 \\
12,29\end{array}$ & $\begin{array}{l}34,48 \\
51,72 \\
13,80\end{array}$ & $\begin{array}{l}33,89 \\
50,84 \\
15,27\end{array}$ & $\begin{array}{l}33,33 \\
50,00 \\
16,67\end{array}$ \\
\hline $\begin{array}{l}30 \\
70\end{array}$ & $\begin{array}{r}29,41 \\
68,62 \\
1,97\end{array}$ & $\begin{array}{r}28,84 \\
67,30 \\
3,86\end{array}$ & $\begin{array}{r}28,30 \\
66,03 \\
5,67\end{array}$ & $\begin{array}{r}27,77 \\
64,81 \\
7,42\end{array}$ & $\begin{array}{r}27,27 \\
63,63 \\
9,10\end{array}$ & $\begin{array}{l}26,48 \\
62,50 \\
10,72\end{array}$ & $\begin{array}{l}26,31 \\
61, n 0 \\
12,29\end{array}$ & $\begin{array}{l}25,86 \\
60,34 \\
13,80\end{array}$ & $\begin{array}{l}25,42 \\
59,32 \\
15,26\end{array}$ & $\begin{array}{l}25,00 \\
58,33 \\
16,67\end{array}$ \\
\hline $\begin{array}{l}20 \\
80\end{array}$ & $\begin{array}{r}19,61 \\
78,43 \\
1,96\end{array}$ & $\begin{array}{r}19,23 \\
76,92 \\
3,85\end{array}$ & $\begin{array}{r}18,86 \\
75,47 \\
5,67\end{array}$ & $\begin{array}{r}18,52 \\
74,07 \\
7,41\end{array}$ & $\begin{array}{r}18,18 \\
72,72 \\
9,10\end{array}$ & $\begin{array}{l}17,86 \\
71,43 \\
10,71\end{array}$ & $\begin{array}{l}17,54 \\
70,17 \\
12,28\end{array}$ & $\begin{array}{l}17,24 \\
68,96 \\
13,80\end{array}$ & $\begin{array}{l}16,95 \\
67,79 \\
15,26\end{array}$ & $\begin{array}{l}16,67 \\
66,66 \\
16,67\end{array}$ \\
\hline $\begin{array}{l}10 \\
90\end{array}$ & $\begin{array}{r}9,80 \\
88,23 \\
1,97\end{array}$ & $\begin{array}{r}9,61 \\
86,53 \\
3,86\end{array}$ & $\begin{array}{r}9,61 \\
84,90 \\
5,49\end{array}$ & $\begin{array}{r}9,25 \\
83,33 \\
7,42\end{array}$ & $\begin{array}{r}9,10 \\
81,80 \\
9,10\end{array}$ & $\begin{array}{r}8,92 \\
80,35 \\
10,73\end{array}$ & $\begin{array}{r}8,77 \\
78,94 \\
12,29\end{array}$ & $\begin{array}{c}8,62 \\
77,58 \\
13,80\end{array}$ & $\begin{array}{r}8,47 \\
76,27 \\
15,26\end{array}$ & $\begin{array}{r}8,33 \\
75,00 \\
16,67\end{array}$ \\
\hline
\end{tabular}

cemento" hay que interpretar, según la tabla mencionada, que la proporción porcentual real de dicha probeta es: $71,43 \%$ de tierra, $17,86 \%$ de arena normalizada y $10,71 \%$ de cemento.

\section{ENSAYOS REALIZADOS: ANALISIS DE RESULTADOS}

\subsection{Introducción}

Dos han sido los datos estudiados en todos los casos: resistencia a compresión y a flexotracción. En ambos casos se procedió sobre probetas de $4 \times 4 \times 16 \mathrm{~cm}$ realizadas en la forma que se describe en el Apartado 2. Al cabo de un día, en ambiente de laboratorio, las probetas se desmoldaban, se pesaban y se mantenían seis días en la cámara de curado a temperatura $\left(21^{\circ} \mathrm{C} \pm 1^{\circ} \mathrm{C}\right)$ y humedad (95\% $\pm 3 \%$ ) constante, pasando seguidamente - durante 24 horas - a una estufa a $60^{\circ} \mathrm{C}$ y $0 \%$ H.R.; transcurrido este tiempo se pesaban nuevamente y se sometían
Table: $71.43 \%$ earth, $17.86 \%$ standardized sand, and $10.71 \%$ cement.

\section{TESTS CARRIED OUT: AN ANALYSIS OF THE RESULTS}

\subsection{Introduction}

Two data were studied in each case: compressive strength and bending tensile strength. In both cases, the tests were carried out upon test pieces produced in the manner described in Part 2. After one day, the test pieces were broken and the pieces weighed. They were then kept for 6 days in a curing chamber at a constant temperature

$\left(21^{\circ} \mathrm{C} \pm 1^{\circ} \mathrm{C}\right)$ and humidity $(95 \% \pm 3 \%)$. Immediately afterwards they were put in a stove for 24 hours at $60^{\circ} \mathrm{C}$ and $0 \%$ R.H. Once the 24 hours was up they were weighed once more and submitted to the bending-tensile 
a ensayo de flexotracción; los dos trozos de probeta resultante se rompian a compresión de ambos valores se presenta el valor de la media, como resultado del ensayo a compresión.

En los gráficos siguientes se representan los tres valores resultantes en cada serie y el valor de la media de éstas.

Otros resultados sobre el comportamiento de este tipo de suelo, han sido publicados por los autores (4) y (5), anteriormente.

\subsection{Influencia del incremento de cemento sobre tierra}

Se prepararon series de probetas a base de tierra, a las que posteriormente se agregaron cantidades crecientes de cemento PA-350, desde $0 \%$ a $20 \%$, en incrementos de $2 \%$ en $2 \%$. Por tratarse de composiciones binarias, los valores porcentuales teóricos y reales son, en este caso, coincidentes.

Los valores medios a compresión y flexotracción, se recogen en la Figura 5. Se deducen las conclusiones siguientes:

I.a. La adición de un $2 \%$ de cemento tiene sobre las dos resistencias mecánicas medidas una influencia nefasta: la compresión cae de 9,0 $\mathrm{MPa}$ a 5,7 $\mathrm{MPa}$ y la flexotracción de 2,23 MPa a 1,76 MPa.

I.b. La tendencia general de caída de resistencias a compresión, sólo se frena para proporciones de cemento superiores al $14 \%$, que equivale en valor real al $12,29 \%$.

I.c. Resulta notable la escasa influencia del cemento sobre la resistencia a flexotracción, que se mantiene entre $0,8 \mathrm{MPa}$ y $1,7 \mathrm{MPa}$, prácticamente para contenidos desde el $2 \%$ de cemento al $20 \%$.

\subsection{Influencia del incremento de cemento sobre tierra/arena}

Partiendo de una proporción constante de tierra $(80 \%)$ y arena normalizada $(20 \%)$, se han realizado series de tres probetas con la incorporación de cemento PA-350, en proporciones crecientes de $2 \%$ en $2 \%$, desde el $0 \%$ al $20 \%$ (para los valores reales, ver Tabla 6). En la figura 6 se recogen los valores medios de las resistencias a compresión y a flexotracción. strength test. The two resulting pieces were then broken by compression. The compressive strength shown is the average of the two values obtained.

The following graphics show the three results of each series and their average.

Other results concerning the behaviour of this type of earth have been previously published by authors (4) (5).

\subsection{Effect of Increases of Cement on Earth}

A series of earth test pieces were prepared to which increasing quantities of $P A-350$ cement were later added. This quantity ranged from $0 \%$ to $20 \%$, and was added in increments of $2 \%$. Since these are binary compositions, in this case the real and theoretical percentages are coincidents.

Figure 5 shows average compressive and bending-tensile strengths obtained. From these results we can deduce the following:

I.a. The addition of $2 \%$ of cement has an extremely negative effect on mechanical strengths: compressive strength falls from 9.0 MPa to 5.7 MPa, and bending-tensile from 2.23 $\mathrm{MPa}$ to $1.76 \mathrm{MPa}$.

I.b. The general trend of a drop in compressive strengths is only stopped at proportions of cement superior to $14 \%$, equivalent to a real value of $12.29 \%$

I.c. The small effect of cement on bendingtensile strength is worth noting. This practically remains within the $0.8 \mathrm{MPa}$ to 1.7 $\mathrm{MPa}$ range for cement contents between $2 \%$ and $20 \%$.

\subsection{Effect of Increases in Cement on Earth/Sand}

A series of three test pieces were prepared using a constant proportion of earth (80\%) and sand (20\%). PA-350 cement was added in increments of $2 \%$, from $0 \%$ to $20 \%$ (real values are given in Table 6). Figure 6 shows the average compressive and bending-tensile strengths. 


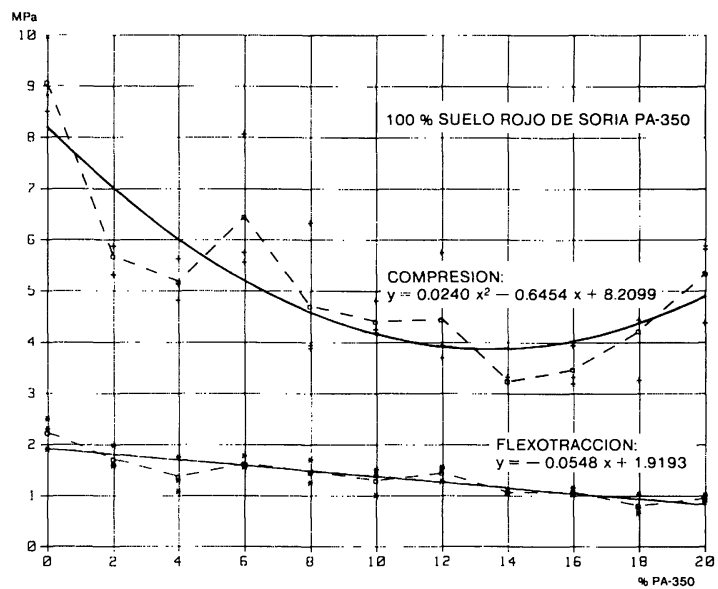

Figura 5

Del análisis de los resultados pueden deducirse algunas conclusiones:

II.a. Las resistencias a compresión y flexotracción máximas se consiguen para la proporción tierra (80 \%), arena (20\%), cemento (0\%).

II.b. La adición de un $2 \%$ de cemento provoca pérdidas superiores al $50 \%$ en ambas resistencias. Para valores del $4 \%$ de cemento las resistencias continúan bajando, aunque en menor proporción.

II.c. Para valores crecientes en la proporción de cemento, desde el $6 \%$ al $20 \%$, no se detectan tendencias claras, aunque puede apuntarse una cierta estabilización de las resistencias a flexotracción y un comportamiento aleatorio en las de compresión, aunque con oscilaciones entre 3,0 MPa y 4,5 MPa, con excepción del valor correspondiente a las probetas con un $16 \%$ de cemento (valor real $13,8 \%)$. Esta serie se repitió y los resultados siguieron confirmando el aumento detectado.

Ha de tenerse presente, a efectos prácticos, que una proporción de cemento del $6 \%$, es decir, del $5,67 \%$ real, supone un consumo aproximado de $118 \mathrm{~kg}$ por metro cúbico de tierra estabilizada seca (suponiendo un peso específico aproximado de $1.980 \mathrm{~kg} / \mathrm{m}^{3}$ ) y que la adición de un $12 \%(10,71 \%$ real) representa $212 \mathrm{~kg}$ de cemento, lo que equivale a un consumo, en cierto modo, comparable al de dosificaciones típicas de hormigones pobres.

Ley de relación compresión-flexotracción:

Para los valores medios de las once series de probetas estudiadas, la figura 7 recoge la recta de ajuste $y=0,26076 x-0,05237$.

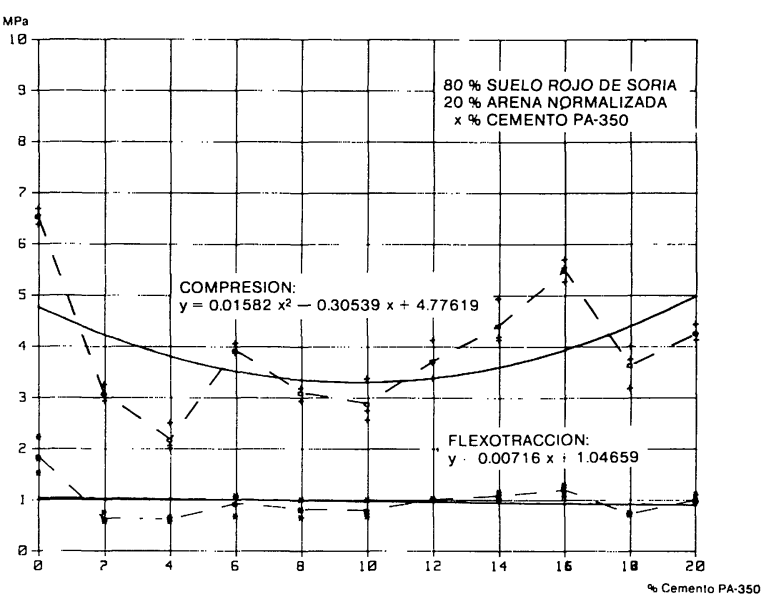

Figura 6

The following can be deduced from an analysis of the results:

II.a. Maximum compressive and bendingtensile strengths are obtained with the proportion earth $80 \%$, sand $20 \%$, and cement $0 \%$.

1I.b. The addition of $2 \%$ of cement causes a drop of over $50 \%$ in both strengths. For $4 \%$ cement, the strengths continue to fall but at a proportionally lower rate.

II.c. No clear trend can be seen for higher cement proportions-from $6 \%$ to $20 \%$ although a certain stabilization in bending-tensile strengths is noted, and a contingent behaviour in compressive ones. These nonetheless oscillate between 3.0 MPa and 4.5 MPa, except for the value corresponding to test pieces containig $16 \%$ cement (real value, $13.8 \%)$. This series was repeated, the results confirming the increase detected.

We must keep in mind, for practical purposes, that a cement proportion of $6 \%$, a real value of $5,6 \%$, means an approximate consumption of $118 \mathrm{~kg} / \mathrm{m}^{3}$ of stabilized dry earth (a specific gravity of approximately $1980 \mathrm{~kg} / \mathrm{m}^{3}$ ). The addition of $12 \%$ cement $(10.71 \%$ real) represents $212 \mathrm{~kg}$ of cement, a consumption somewhat comparable with normal mixes of poor concretes.

The Compression-Bending-Tensile Relation Law

Figure 7 shows the line $y=0.26076 x=0.05237$ for the average values of the eleven series of test pieces studied. 


\subsection{Influencia del tipo de cemento}

Con objeto de detectar y cuantificar la influencia del tipo de cemento sobre las características mecánicas: compresión y flexotracción, se procedió a realizar series de probetas a base de distintas proporciones tierra/arena, con un $12 \%$ de cemento en idénticas condiciones y utilizando dos tipos de cemento: PA-350 y P-450. El primero de ellos es un cemento portland que puede tener hasta un $20 \%$ de adiciones activas, y el segundo es un portland normal.

En la figura 8 se recogen los resultados a compresión y flexotracción de ambas series, de los que se deducen las conclusiones siguientes:

III.a. La influencia del tipo de cemento empleado es francamente notable; en algunos casos se superan los incrementos del $100 \%$ al emplear el P-450 respecto del PA-350.

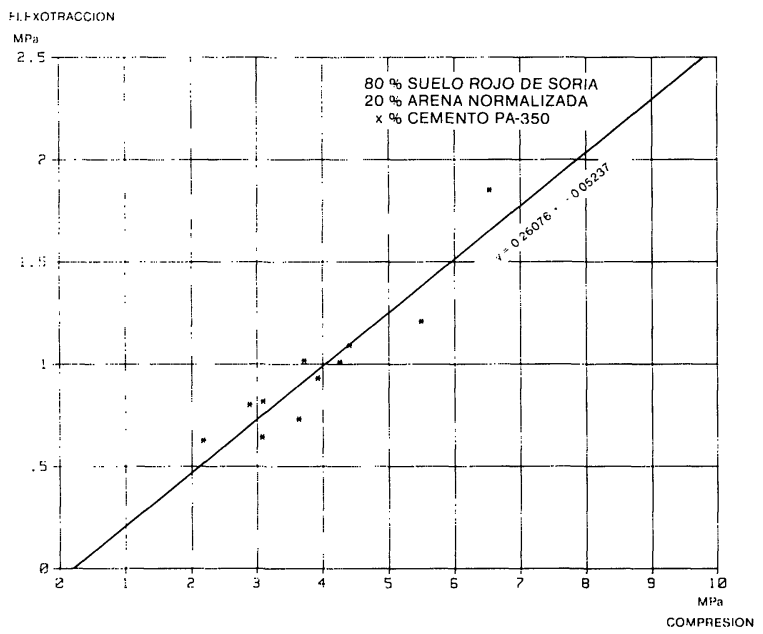

Figura 7

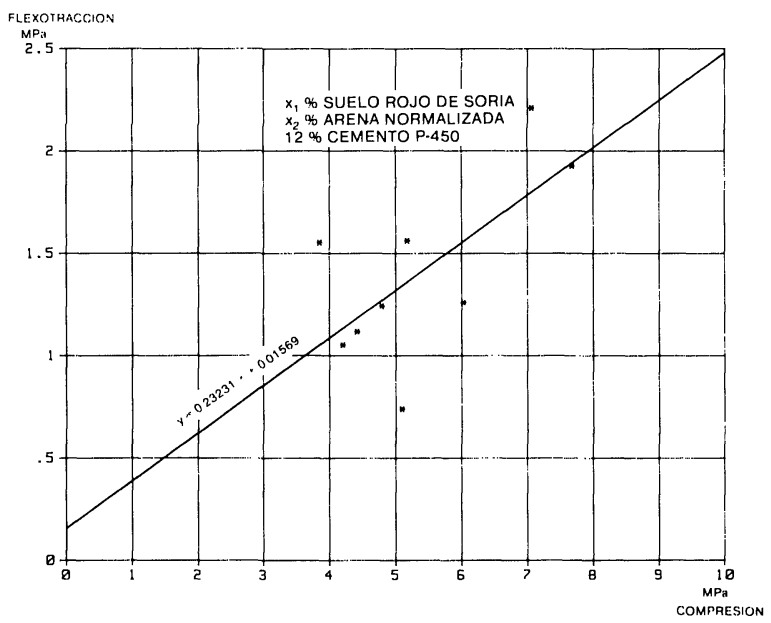

Figura 9

\subsection{Effect of the Type of Cement}

A series of test pieces containing different earth/sand proportions and $12 \%$ cement were prepared in identical conditions in order to determine the effect of two different cements, $P A-350$ and $P-450$, on mechanical strengths. The first cement is a Portland cement capable of containing up to $20 \%$ active additions. The second is a normal Portland cement.

Figure 8 shows the compressive and bending-tensile results for both series, from which we deduce the following:

III.a. The effect of the type of cement employed is worth noting. In some cases, increases of more than $100 \%$ are seen in the use of $P-450$ with respect to PA-350.

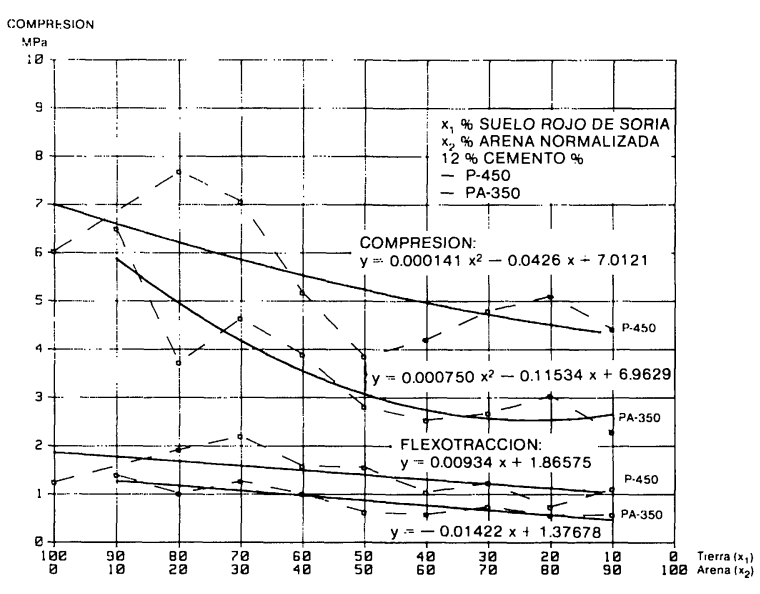

Figura 8

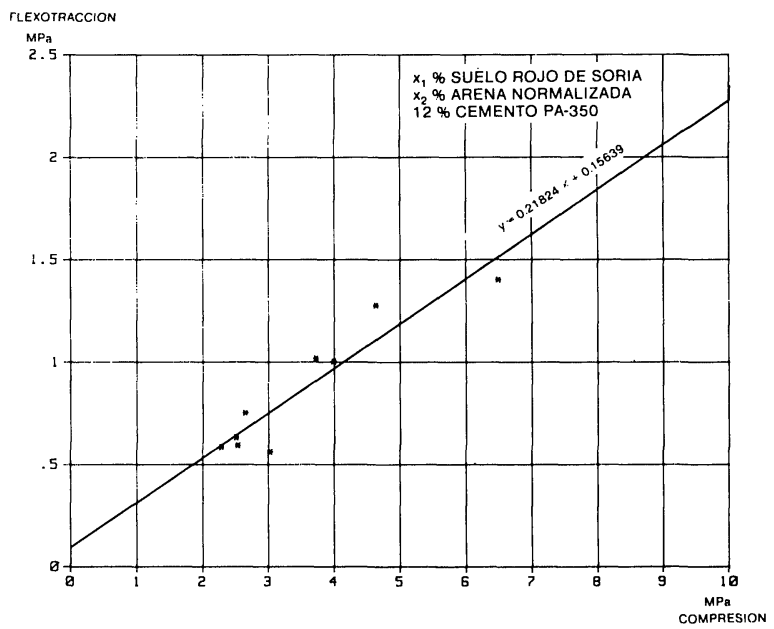

Figura 10

MATERIALES DE CONSTRUCCION, Vol. 38, n. 210 , abril/mayo/junio 1988 
III.b. Para la tierra y arena empleadas, la proporción tierra/arena comprendidas en la zona $80 / 20 \div 60 / 40$ se muestran las más adecuadas, tanto a compresión como a flexotracción, y ello para los dos tipos de cementos considerados.

III.c. Las figuras 9 y 10 muestran la muy clara relación existente entre la resistencia a la compresión y a flexotracción en ambas series de probetas.

\subsection{Variaciones tierra/arena/cemento}

Se prepararon series de probetas, con la metodología descrita en el Apartado 2, de relaciones porcentuales tierra/arena normalizada: $80 \%-20 \% ; 70 \%-30 \%$; $60 \%-40 \% ; 50 \%-50 \% ; 40 \%-60 \%$; $30 \%-70 \% ; 20 \%-80 \%$ y $10 \%-90 \%$.

Cada una de estas series se repitió con adiciones de cemento PA-350 de $0 \% ; 2 \%$; $6 \% ; 12 \%$ y $20 \%$, lo que supone un total de 120 probetas ensayadas.

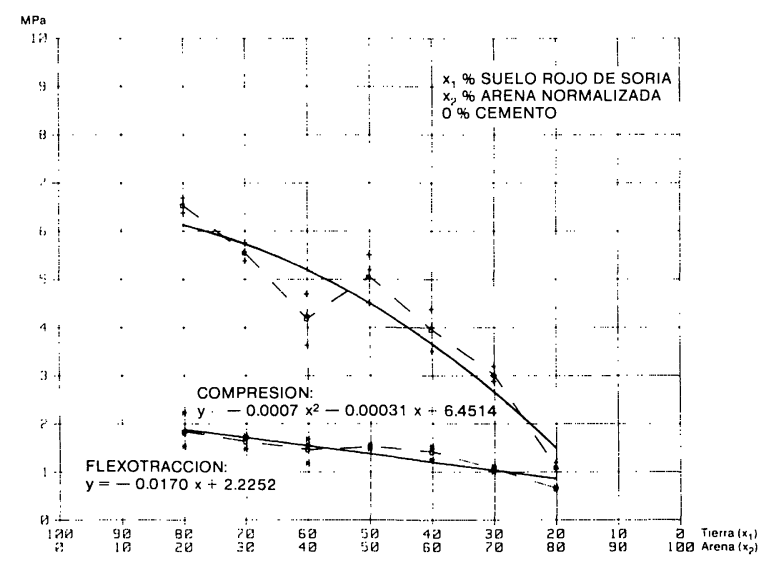

Figura 11

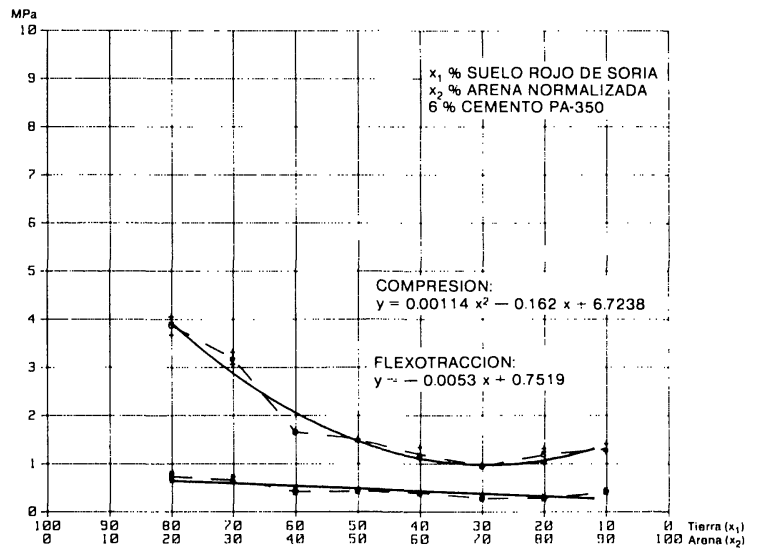

Figura 13
III.b. With respect to the earth and sand used, the earth/sand proportion between $80 / 20$ and 60/40 appears most adequate, both for compressive stength as well as bending-tensile, and this for both types of cement considered.

III.c. The clear relation between compressive and bending-tensile strengths in both series is seen in Fig. 9 and 10.

\subsection{Earth/Sand/Cement Variations}

Series of test pieces were prepared as in Part 2 of the following earth/standardized sand percentages: $80 / 20,70 / 30,60 / 40,50 / 50$, $40 / 60,30 / 70,20 / 80$, and 10/90.

Each series was repeated with cement additions of $0 \%, 2 \%, 6 \%, 12 \%$, and $20 \%$. A total of 120 test pieces were tested.

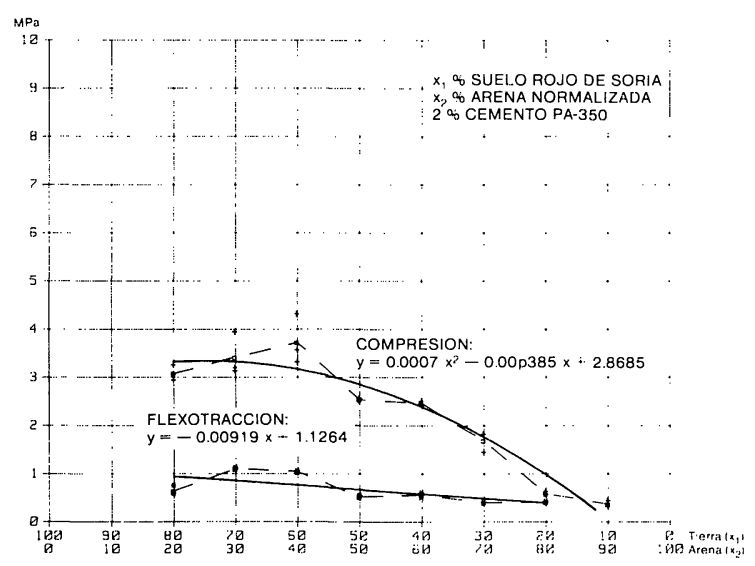

Figura 12

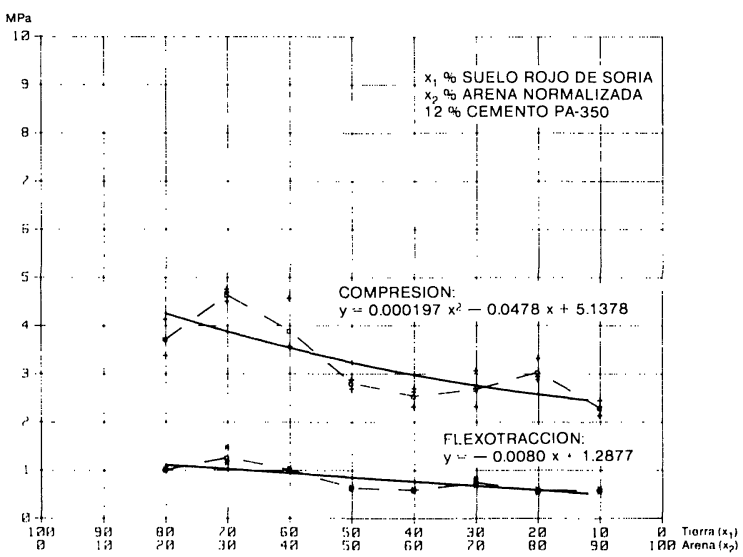

Figura 14 
Los valores medios de las series a compresión y flexotracción se recogen en las figuras 11 a 15. De las figuras aludidas se desprenden las siguientes conclusiones:

IV.a. Las series de probetas sin cemento presentan caídas de resistencias, tanto a compresión como a flexotracción, conforme aumenta la proporción de arena normalizada.

IV.b. La adición del $2 \%$ de cemento no frena la caída de la resistencia aludida, sólo que con valores sensiblemente inferiores a los del caso anterior.

IV.c. La aportación del cemento en una proporción teórica del $12 \%$ frena las pérdidas de resistencia sólo para proporciones tierra/arena iguales o menores de 50/50, lo cual es explicable por el comportamiento como mortero de la arena normalizada con el cemento. La tierra en estos casos tiene una presencia perturbadora.

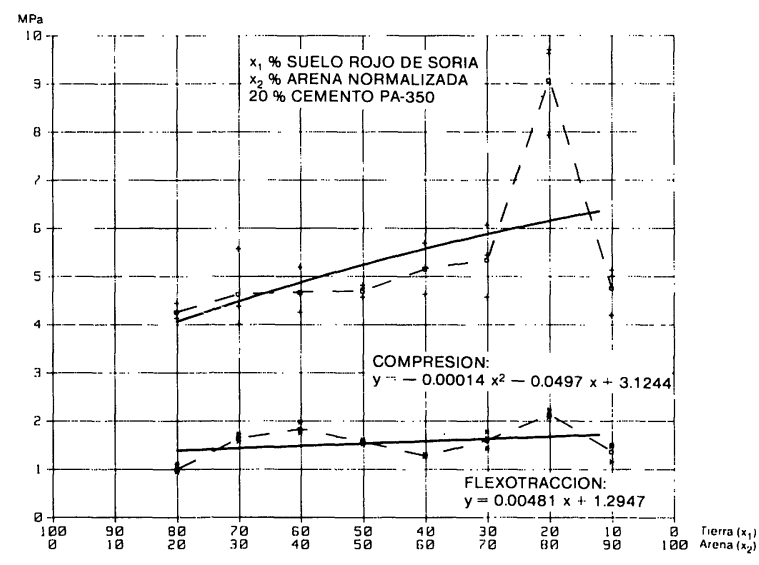

Figura 15

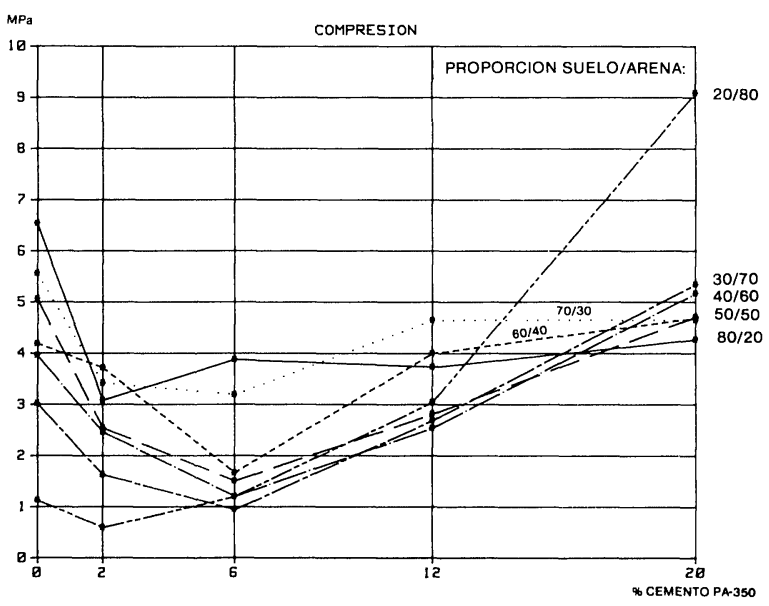

Figura 17
The average compressive and bending-tensile strengths are shown in Figs. 11 to 15. We can draw the following conclusions from this data:

IV.c. The series of test pieces without cement show a drop in strength, both compressive and bending-tensile, as the percentage of standardized sand increases.

IV.b. The addition of $2 \%$ cement does not stop the above-mentioned fall, although this drop is not as steep as in IV.a.

IV.c. The addition of $12 \%$ cement (theoretical proportion) stops loss of strength only for earth/sand mixed equal to or less than $50 / 50$, a fact explained by the behaviour as a mortar of standardized sand with cement. In these cases, earth has a perturbing effect.

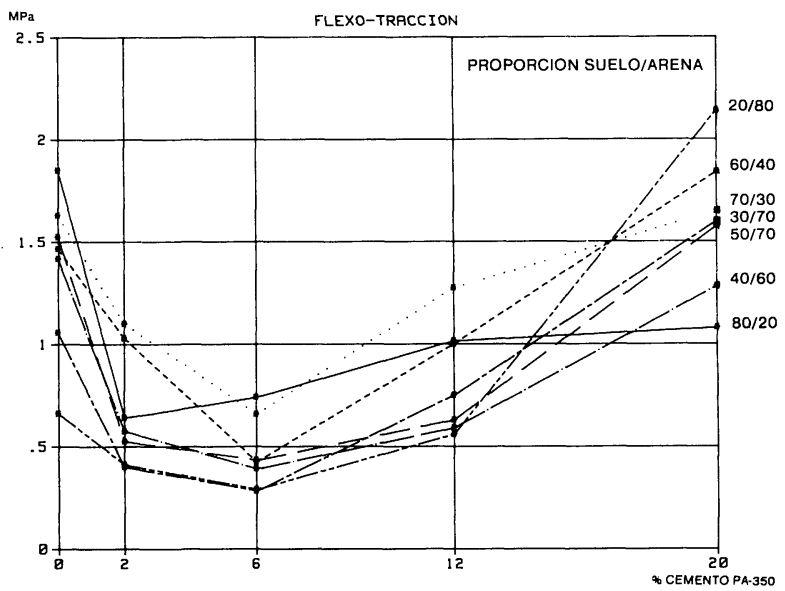

Figura 16

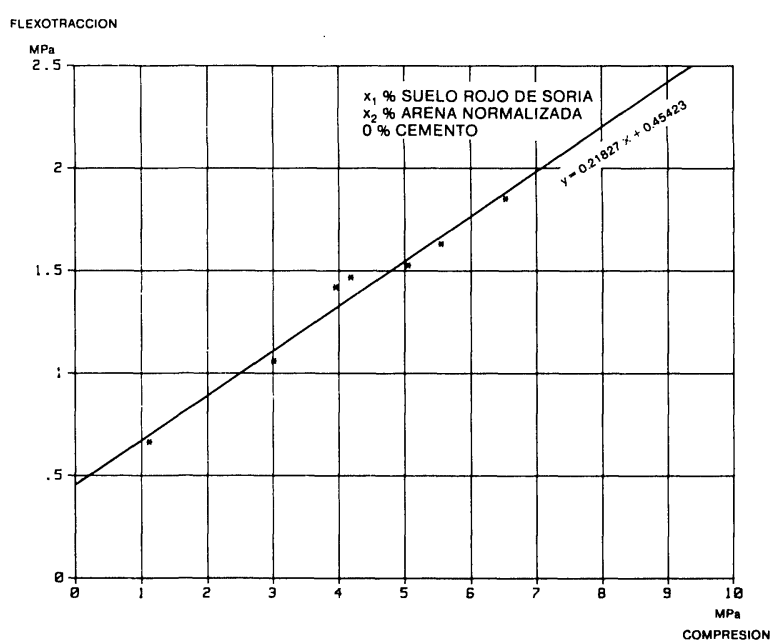

Figura 18 
IV.d. La adición de un $20 \%$ teórico de cemento, lo que equivale a unos $330 \mathrm{~kg}$ de cemento por metro cúbico (proporción que hace que el resultado se considere más como "geo-hormigón" u hormigón de tierra que como tierra propiamente), sólo consigue que prácticamente se mantenga estable la resistencia a compresión, entre 4,0 MPa y 5,5 $\mathrm{MPa}$ para toda la serie. Las figuras 16 y 17 recogen con nitidez - para flexotracción la primera y para compresión la segunda- el conjunto de los resultados representados en las figuras 11 a 15 inclusive, sólo que representando en abscisas los valores porcentuales de cemento aportados.

De forma general, para la tierra ensayada, puede afirmarse de acuerdo con la figura 16 que:

V.a. Se detectan caídas importantes a flexotracción para adiciones del $2 \%$ de cemento, que siguen decreciendo para el $6 \%$.

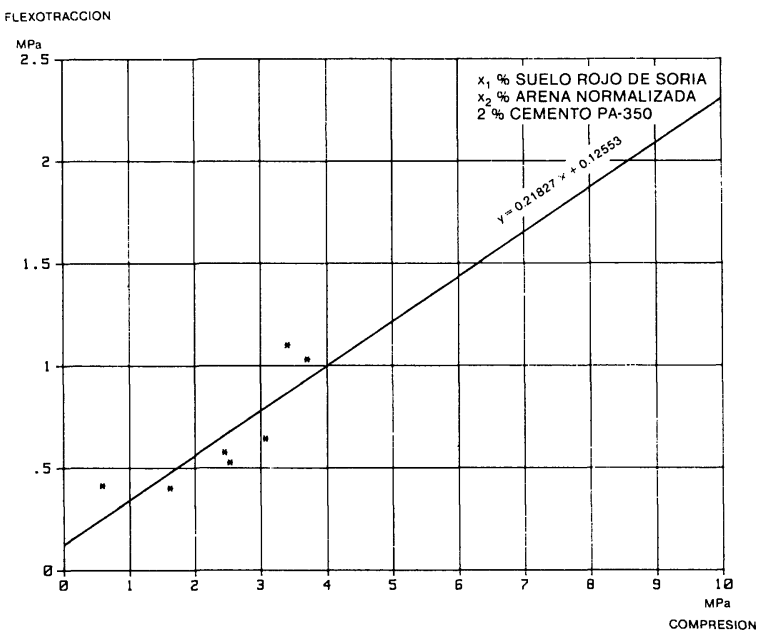

Figura 19

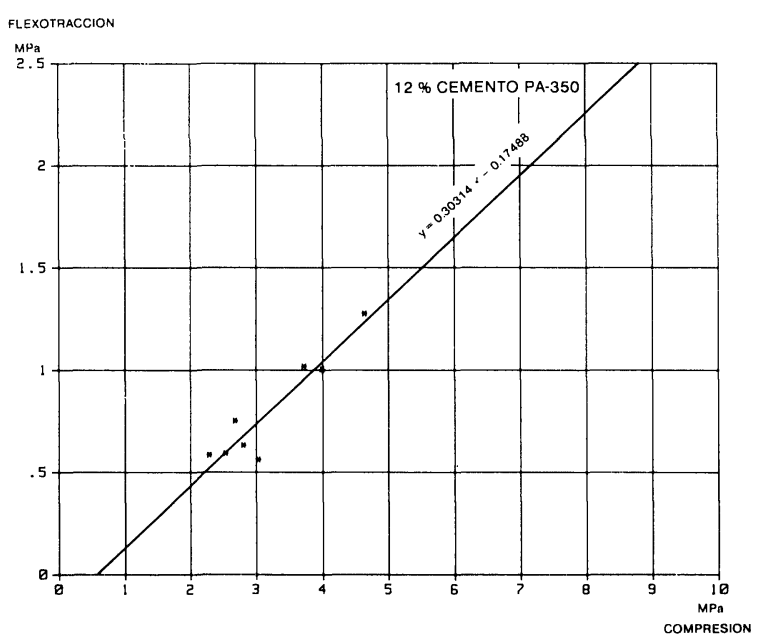

Figura 21
IV.d. The addition of $20 \%$ theoretical of cement, equivalent to $330 \mathrm{~kg} / \mathrm{m}^{3}$ (a proportion which makes us consider this mix as a "geoconcrete" rather than earth) only manages to maintain the values practically stable, between 4.0 MPa and 5.5 MPa, for all the series. Figures 16 and 17 show clearly, bending-tensile the first and compressive the second, the set of results shown in Figs. 11 to 15, with the percentage of cement added plotted on the abscissas.

In general and with respect to the earth tested, the following can be deduced from Fig. 16:

V.a. The addition of $2 \%$ of cement yields important drops in bending-tensile strengths, which continues falling at $6 \%$.

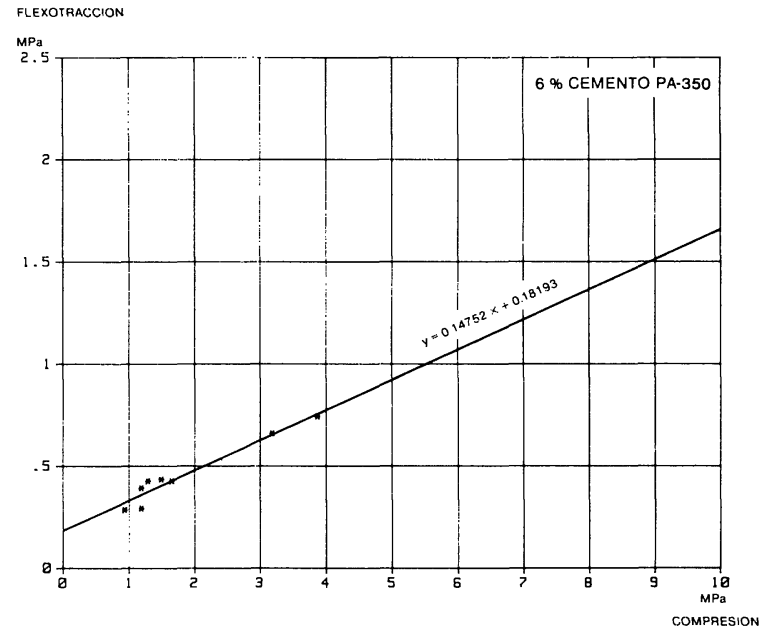

Figura 20

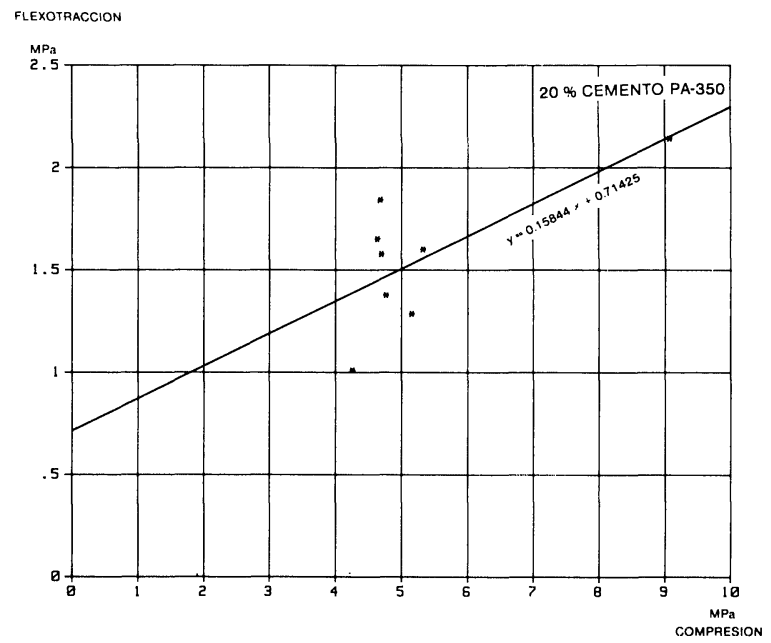

Figura 22 
V.b. Al pasar del $6 \%$ de cemento al $12 \%$ y, en mayor proporción, al $20 \%$, se detecta una recuperación creciente de los resultados a compresión.

V.c. Resulta necesaria la adición de un $20 \%$ de cemento para conseguir resultados comparables a los que proporciona sólo el suelo.

De los resultados a compresión (figura 17), se deduce:

VI.a. Decrecen sensiblemente los resultados al añadir un $2 \%$ de cemento PA-350. Continúan decreciendo al pasar a un $6 \%$ de cemento, con excepción de las proporciones tierra/arena del $80 / 20$ y del 20/80.

VI.b. Todas las proporciones tierra/arena ven incrementar, de forma sensible y cuasi en forma lineal, sus resistencias a compresión, cuando se pasa del $6 \%$ al $12 \%$ de cemento.

VI.c. Se detectan crecimientos moderados en todos los casos, excepto en la proporción tierra/arena de $20 / 80$, que es del orden del $200 \%$, al incrementar el cemento del $12 \%$ al $20 \%$.

En las figuras 18 a 22 inclusive, se representan las correlaciones entre las resistencias a compresión y flexotracción de los valores recogidos en las figuras 11 a 15 respectivamente.
V.b. Going from $6 \%$ to $12 \%$ and, in to a grater extent at $20 \%$, an increasing recovery of compressive results is seen.

V.c. It is necessary to add $20 \%$ cement in order to obtain results similar to those of earth alone.

And with respect to compressive stengths, from Fig. 17 we can deduce:

VI.a. The results decrease notably upon adding $2 \%$ of PA-350. They continue falling until $6 \%$, with the exception of earth/sand mixes of $80 / 20$ and 20/80.

VI.b. All earth/sand proportions show an increase in compressive strengths, almost in a linear fashion, going from $6 \%$ to $12 \%$ cement.

VI.c. Moderate increases are seen in all cases as the cement proportion increases from $12 \%$ to $20 \%$, except for earth/sand $20 / 80$, where it is around $200 \%$.

Figs. 18 to 22 show the correlations between compressive and bending-tensile strengths for the values shown in Figs. 11 to 15 respectively.

\section{REFERENCIAS BIBLIOGRAFICAS}

(1) CRATerre: "Construire en Terre". Editions Alternatives, pp. 285. París, 1979.

(2) Department of Scientific and Industrial: Traducción de L. Valero. "Mecánica del Suelo". Centro de Estudios y Experimentación de Obras Públicas. pp. 659. Madrid, 1963.

(3) BRANSAKA, Muhirve: "Habitat au Burundi: l'apport du matériau terre". pp. 99-112. UCL-UCO, 1982.

(4) GUINEA, María Jesús: "La tierra material resistente al agua". Monografía n.॰385/386. Instituto Eduarto Torroja. pp. 25-30. 1987.

(5) SALAS, Julián; GUINEA, M. a Jesús; NAREDO, Teresa: "Influencia de cinco procesos de curado en las resistencias mecánicas de probetas de tierra". (Pendiente de su publicación). Housing Science and its applications. 